\title{
Origins of genuine Ohmic van der Waals contact between indium and $\mathrm{MoS}_{2}$
}

Bum-Kyu Kim ${ }^{1,7}$, Tae-Hyung Kim ${ }^{2,7}$, Dong-Hwan Choi $\mathbb{D}^{1,3,7}$, Hanul Kim ${ }^{3}$, Kenji Watanabe $\mathbb{D}^{4}$, Takashi Taniguchi ${ }^{5}$, Heesuk Rho ${ }^{3}$,

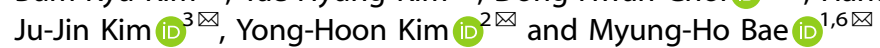

The achievement of ultraclean Ohmic van der Waals (vdW) contacts at metal/transition-metal dichalcogenide (TMDC) interfaces would represent a critical step for the development of high-performance electronic and optoelectronic devices based on twodimensional (2D) semiconductors. Herein, we report the fabrication of ultraclean vdW contacts between indium (In) and molybdenum disulfide $\left(\mathrm{MoS}_{2}\right)$ and the clarification of the atomistic origins of its Ohmic-like transport properties. Atomically clean $\mathrm{In} / \mathrm{MoS}_{2}$ vdW contacts are achieved by evaporating In with a relatively low thermal energy and subsequently cooling the substrate holder down to $\sim 100 \mathrm{~K}$ by liquid nitrogen. We reveal that the high-quality $\mathrm{In} / \mathrm{MoS}_{2} \mathrm{vdW}$ contacts are characterized by a small interfacial charge transfer and the Ohmic-like transport based on the field-emission mechanism over a wide temperature range from 2.4 to $300 \mathrm{~K}$. Accordingly, the contact resistance reaches $\sim 600 \Omega \mu \mathrm{m}$ and $\sim 1000 \Omega \mu \mathrm{m}$ at cryogenic temperatures for the fewlayer and monolayer $\mathrm{MoS}_{2}$ cases, respectively. Density functional calculations show that the formation of large in-gap states due to the hybridization between In and $\mathrm{MoS}_{2}$ conduction band edge states is the microscopic origins of the Ohmic charge injection. We suggest that seeking a mechanism to generate strong density of in-gap states while maintaining the pristine contact geometry with marginal interfacial charge transfer could be a general strategy to simultaneously avoid Fermi-level pinning and minimize contact resistance for $2 \mathrm{D}$ vdW materials.

npj 2D Materials and Applications (2021)5:9; https://doi.org/10.1038/s41699-020-00191-z

\section{INTRODUCTION}

Layered semiconducting transition-metal dichalcogenides (TMDCs) such as $\mathrm{MoS}_{2}$, WSe $\mathrm{W}_{2}$, and $\mathrm{MoTe}_{2}$ have been extensively studied for the future development of low-power and highperformance electronic and optoelectronic applications ${ }^{1-3}$. However, establishing a reliable Ohmic contact between metals and TMDCs remains a critical challenge ${ }^{4,5}$. For instance, in reducing the Schottky barrier height $\left(\phi_{\mathrm{SB}}\right)$ for TMDCs, efforts to identify metals with appropriate work functions $\Phi_{\text {metal }}$ (e.g. $\Phi_{\mathrm{Sc}} \approx 3.5 \mathrm{eV}$ for Sc and $\Phi_{\mathrm{Ti}} \approx 4.3 \mathrm{eV}$ for Ti) based on the electron affinity of monolayer (1L) and few-layer TMDCs (e.g. monolayer $\mathrm{MoS}_{2}: \mathrm{X}_{1 \mathrm{LMoS}} \approx 4 \mathrm{eV}$, multilayer $\mathrm{MoS}_{2}: \mathrm{X}_{\mathrm{MLMOS}_{2}} \approx 4.3 \mathrm{eV}$ ) have not been effective because of the strong Fermi-level pinning (FLP) effect ${ }^{6,7}$. While various approaches have been explored to overcome this problem, including molecular doping ${ }^{8}$, tunnel-barrier insertion ${ }^{9,10}$, fabrication of graphene contacts ${ }^{11,12}$, and TMDC phase changes ${ }^{13^{\prime}}$, recent studies have shown that the formation of an ideal or defect-free metal-TMDC van der Waals (vdW) contact through the transfer of atomically flat metal thin films significantly improves the contact properties ${ }^{14-16}$. In these advances, it was important to recognize that the conventional thermal evaporation process of metals typically introduces crystalline defects in TMDCs and leads to an uncontrollable $\phi_{\mathrm{SB}}$ (or FLP) and high contact resistance ${ }^{7,14}$. Meanwhile, several groups overcame the challenge and showed that the thermal-evaporation process of indium (In) can lead to clean vdW-type contacts for TMDCs with very low contact resistance values ${ }^{17-19}$. However, regarding our initial report on the achievement of Ohmic-like three-dimensional (3D) In-2D $\mathrm{MoS}_{2}$ $\operatorname{contact}^{18}$, question was raised in that the origins of the low contact resistance through the In-MoS 2 vdW contact are unclear.

Here, we prepare an ultraclean vdW contact between In and $\mathrm{MoS}_{2}$ using an improved evaporation method, and clarify through extensive transport measurements and computations the nature and origins of Ohmic transport characteristics from In/MoS 2 vdW contacts. We prepare the high-quality vdW In/MoS interface by lowering the temperature $(T)$ of the substrate holder to $\sim 100 \mathrm{~K}$ during the metal deposition process and utilizing the relatively low In evaporation $T$ of $\sim 530^{\circ} \mathrm{C}$. The $\mathrm{In} / \mathrm{MoS}_{2}$ interface is then characterized by the transmission electron microscope (TEM) and Raman measurements as well as density functional theory (DFT) calculations. DFT calculations show that in comparison with the $\mathrm{Au} / \mathrm{MoS}_{2}$ counterpart, the $\mathrm{In} / \mathrm{MoS}_{2}$ contact induces an order of magnitude smaller charge transfer, which is consistent with the Raman spectroscopy data. For both mono- and few-layer $\mathrm{MoS}_{2}$ devices prepared through our approach, we find that the contact resistance decreases with decreasing $T$ from room temperature to $T=100 \mathrm{~K}$ and $2.4 \mathrm{~K}$, reaching to $1 \mathrm{k} \Omega \mu \mathrm{m}$ and $0.6 \mathrm{k} \Omega \mu \mathrm{m}$, respectively. This behavior indicates the Ohmic-like character at the $\mathrm{In} / \mathrm{MoS}_{2}$ contact and the field emission is the dominant charge transport mechanism at the $\mathrm{In} / \mathrm{MoS}_{2}$ contact. We finally identify from DFT calculations large in-gap states originating from the hybridization between $\mathrm{MoS}_{2}$ conduction-band-edge and In Fermilevel states, which provides a mechanism to achieve an Ohmic contact in spite of the marginal interfacial charge transfer.

\footnotetext{
${ }^{1}$ Korea Research Institute of Standards and Science, Daejeon 34113, Republic of Korea. ${ }^{2}$ School of Electrical Engineering, Korea Advanced Institute of Science and Technology, 291 Daehak-ro, Yuseong-gu, Daejeon 34141, Republic of Korea. ${ }^{3}$ Department of Physics, Research Institute of Physics and Chemistry, Jeonbuk National University, Jeonju 54896, Republic of Korea. ${ }^{4}$ Research Center for Functional Materials, National Institute for Materials Science, 1-1 Namiki, Tsukuba 305-0044, Japan. ${ }^{5}$ International Center for Materials Nanoarchitectonics, National Institute for Materials Science, 1-1 Namiki, Tsukuba 305-0044, Japan. ${ }^{6}$ Department of Nano Science, University of Science and Technology, Daejeon

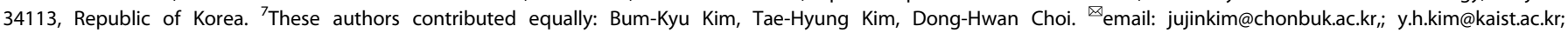
mhbae@kriss.re.kr
} 
a

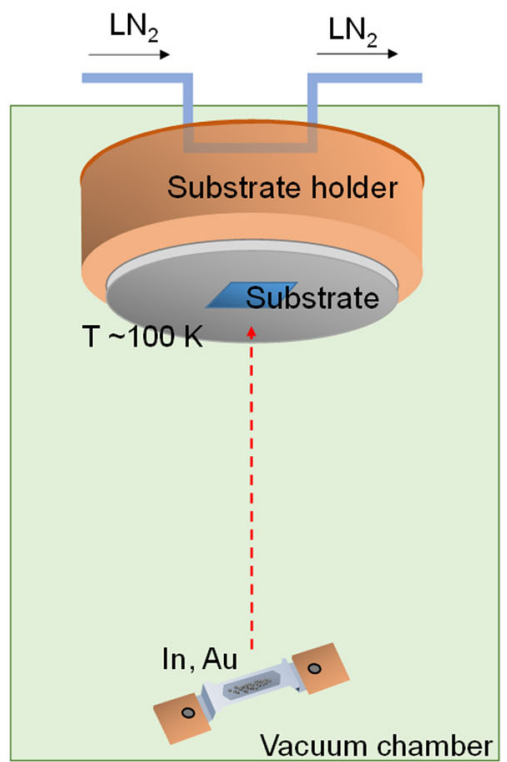

b
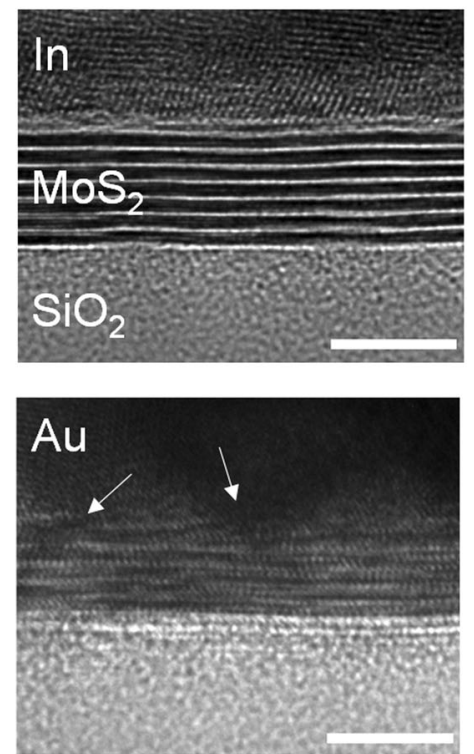

Fig. 1 Metal deposition on $\mathbf{M o S}_{\mathbf{2}}$. a In (Au) evaporation in a vacuum chamber with a liquid-nitrogen cooled sample holder. $\mathbf{b}$ Top and bottom: cross-sectional TEM images of the In/MoS 2 and Au/MoS 2 interfaces, respectively. Scale bar: $5 \mathrm{~nm}$.

\section{RESULTS AND DISCUSSION}

Characterizations of the ultraclean In/MoS 2 interface

We fabricated $\mathrm{MoS}_{2}$ field-effect transistors (FETs) on hexagonal boron nitride $(h$-BN) flakes, where the $h$-BN flakes were deposited onto a $300-\mathrm{nm}$-thick $\mathrm{SiO}_{2} / \mathrm{Si}$ substrate by mechanical exfoliation. We then transferred a few-layer $\mathrm{MoS}_{2}$ flake (HQ-graphene, Inc.) onto a selected $h$-BN flake ${ }^{12,20}$. For the electrical measurements, we deposited 100-nm-thick In electrodes across the $\mathrm{MoS}_{2}$ channel, where the substrate holder was kept at $\sim 100 \mathrm{~K}$ by flowing liquid nitrogen through it (see Fig. 1a). The substrate cooling process leads to an important result, namely a highly uniform surface morphology of In film. This contrasts strongly with the usage of a room-temperature holder that produces a segregated granular film for at least up to $\sim 70 \mathrm{~nm}$ thickness, as reported in our previous work (also see Supplementary Fig. 1) ${ }^{21}$. The upper panel of Fig. $1 \mathrm{~b}$ shows a cross-sectional TEM image of the In/few-layered $\mathrm{MoS}_{2}$ junction, which clearly shows an atomically separated interface between In and $\mathrm{MoS}_{2}$ layers without any metal invasion into the $\mathrm{MoS}_{2}$ layers. Whereas crystal-lattice disorders that cause defect-induced gap states and FLP typically occur during the hightemperature deposition process of evaporated metal atoms with high thermal energy ${ }^{7,14}$, the In deposited at a relatively low thermal energy could apparently provide a clean vdW interface without disorder or defect. For instance, whereas the evaporation temperature of Au at $10^{-7}$ Torr is $\sim 860^{\circ} \mathrm{C}$, only $\sim 530^{\circ} \mathrm{C}$ is required for the evaporation of In at the same pressure. For comparison, we prepared an $\mathrm{Au} / \mathrm{few}$-layered $\mathrm{MoS}_{2}$ junction, where the substrate holder was also kept at $\sim 100 \mathrm{~K}$ during Au deposition. The lower panel of Fig. $1 \mathrm{~b}$ shows a TEM image of the $\mathrm{Au} / \mathrm{MoS}_{2}$ junction, where we observe that the invasion of $\mathrm{Au}$ atoms during the deposition process produces atomic defects at the first and second layers from the $\mathrm{MoS}_{2}$ interface, like previous studies ${ }^{14}$.

To further characterize the quality of In/MoS 2 interface, we applied the Raman spectroscopy for both the pristine and Incovered $\mathrm{MoS}_{2}$ regions to estimate doping effect at the metal contacts. It is known that the $A_{1 \mathrm{~g}}$ phonon peak of $\mathrm{MoS}_{2}$ exhibits a red shift and its width broadens with electron doping ${ }^{22,23}$. Figure $2 \mathrm{a}, \mathrm{b}$ shows the optical images of, respectively, $1 \mathrm{~L}-$ and bilayer (2L)- $\mathrm{MoS}_{2}$ (indicated by regions bounded with dashed black lines) prepared on $\mathrm{SiO}_{2}$ and partially covered with $5-\mathrm{nm}$ thick In (indicated by regions bounded with dashed white lines).
Figure $2 \mathrm{c}$, d shows the $A_{1 \mathrm{~g}}$ energy maps for the $1 \mathrm{~L}$ - and $2 \mathrm{~L}-\mathrm{MoS}_{2}$, respectively. The In-covered region shows a relatively lower energy than the non-covered region, i.e., $\Delta \omega \approx-0.3$ and $-1 \mathrm{~cm}^{-1}$ for the $1 \mathrm{~L}$ - and $2 \mathrm{~L}-\mathrm{MoS}_{2}$, respectively (see Supplementary Fig. 2 for representative Raman spectra for a $\left.1 \mathrm{~L}-\mathrm{MoS}_{2}\right)$. In the upper panel of Supplementary Fig. 2a, we also show the $E_{2 g}^{1}$ energy map of the $1 \mathrm{~L}-\mathrm{MoS}_{2}$, which indicates relatively negligible difference between the In-covered and non-covered regions. For the biaxial strain, the red shift of the $E_{2 g}^{1}$ Raman mode corresponding to the in-plane vibration is more sensitive than the $A_{1 \mathrm{~g}}$ mode (out-of-plane vibration $)^{24,25}$. For the in- and out-of-plane compressive strains, both modes should show blue shifts ${ }^{26}$. In our case, however, we observed a relatively strong red shift of the $A_{1 \mathrm{~g}}$ mode than that of the $E_{2 g}^{1}$ mode, which has been interpreted as a doping effect ${ }^{22}$. For instance, Chakraborty et al. ${ }^{22}$ reported that the $A_{1 \mathrm{~g}}$ mode softens with doping at a rate of $\sim 0.2 \mathrm{~cm}^{-1}$ per $10^{12} \mathrm{~cm}^{-2}$ for $1 \mathrm{~L}-\mathrm{MoS}_{2}$, whereas the $E_{2 g}^{1}$ mode is relatively insensitive to the doping. We thus conclude that the $1 \mathrm{~L}-\mathrm{MoS}_{2}$ region covered by In was doped by electrons at a density of $\sim+1.5 \times 10^{12}$ electrons $\mathrm{cm}^{-2}$ (electron accumulation in $\mathrm{MoS}_{2}$ ). The full-width at half-maximums, $\Gamma$, in Fig. 2e, f also show consistent results. For instance, the In-covered region shows a relatively broader $\Gamma$ than the non-covered region for both $1 \mathrm{~L}-$ and $2 \mathrm{~L}-\mathrm{MoS}_{2}$, implying electron doping. This In-to$\mathrm{MoS}_{2}$ electron transfer feature will be further discussed below based on DFT calculations and shown to be another strong indication of ultraclean vdW contacts.

\section{Charge redistribution at the $\mathrm{In} / \mathrm{MoS}_{2}$ interface}

To extract the atomistic information of the $\mathrm{In} / \mathrm{MoS}_{2} \mathrm{vdW}$ contact and contrast them with those of the $\mathrm{Au} / \mathrm{MoS}_{2}$ counterpart, we carried out DFT calculations for the vertical In/MoS 2 and $\mathrm{Au} /$ $\mathrm{MoS}_{2}$ interface models and applied several analysis methods $^{27,28}$. In Fig. 3a, b top panels, we show the fully optimized In/MoS 2 and Au/MoS 2 contact models, respectively (see details in Supplementary Figs. 3, 4 and Table 1). First, compared to the $\mathrm{Au} / \mathrm{MoS}_{2}$ case, we find in the optimized In/MoS 2 atomic structure negligible structural distortions at the rightmost metal atomic layer (see also Supplementary Fig. 3). As supported by the DFT-estimated binding energy ${ }^{29,30}$ as well as the explicit experimental demonstration for the transferred Au electrode ${ }^{14}$, $\mathrm{Au}$ is a representative metal that forms vdW-type interactions 
a

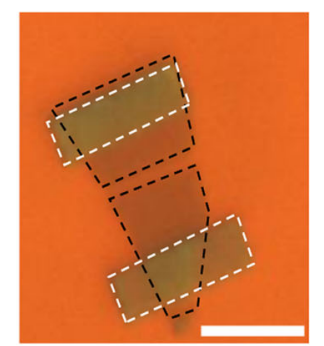

b

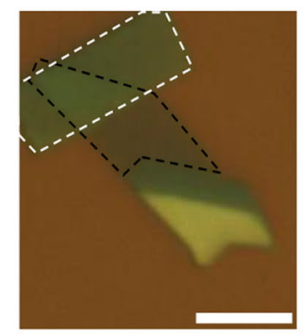

C

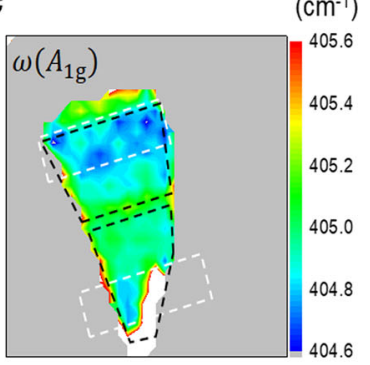

d

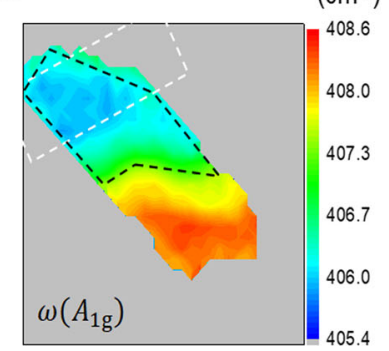

e

$\left(\mathrm{cm}^{-1}\right)$

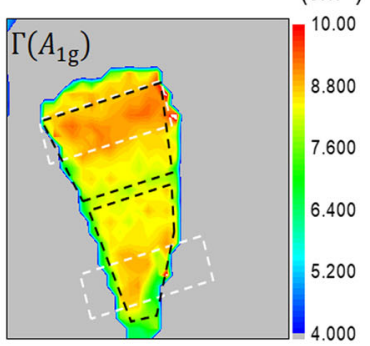

f

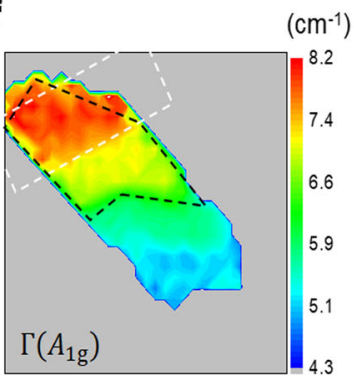

Fig. 2 Raman spectroscopy for $\ln / \mathrm{MoS}_{2}$. a, b Optical images of $1 \mathrm{~L}$ - and $2 \mathrm{~L}-\mathrm{MoS}_{2}$ (dashed black-boxed region) on $\mathrm{SiO}_{2}$ substrates, respectively. White boxed regions: 5 -nm-thick In-deposited regions. Scale bar: $5 \mu \mathrm{m}$. c, d $A_{1 \mathrm{~g}}$ energy $(\omega)$ maps for $1 \mathrm{~L}-$ and $2 \mathrm{~L}-\mathrm{MoS}_{2}$, respectively. e, f Fullwidth at half-maximum ( $\Gamma$ ) maps of $A_{1 \mathrm{~g}}$ for $1 \mathrm{~L}-$ and $2 \mathrm{~L}-\mathrm{MoS}_{2}$, respectively.
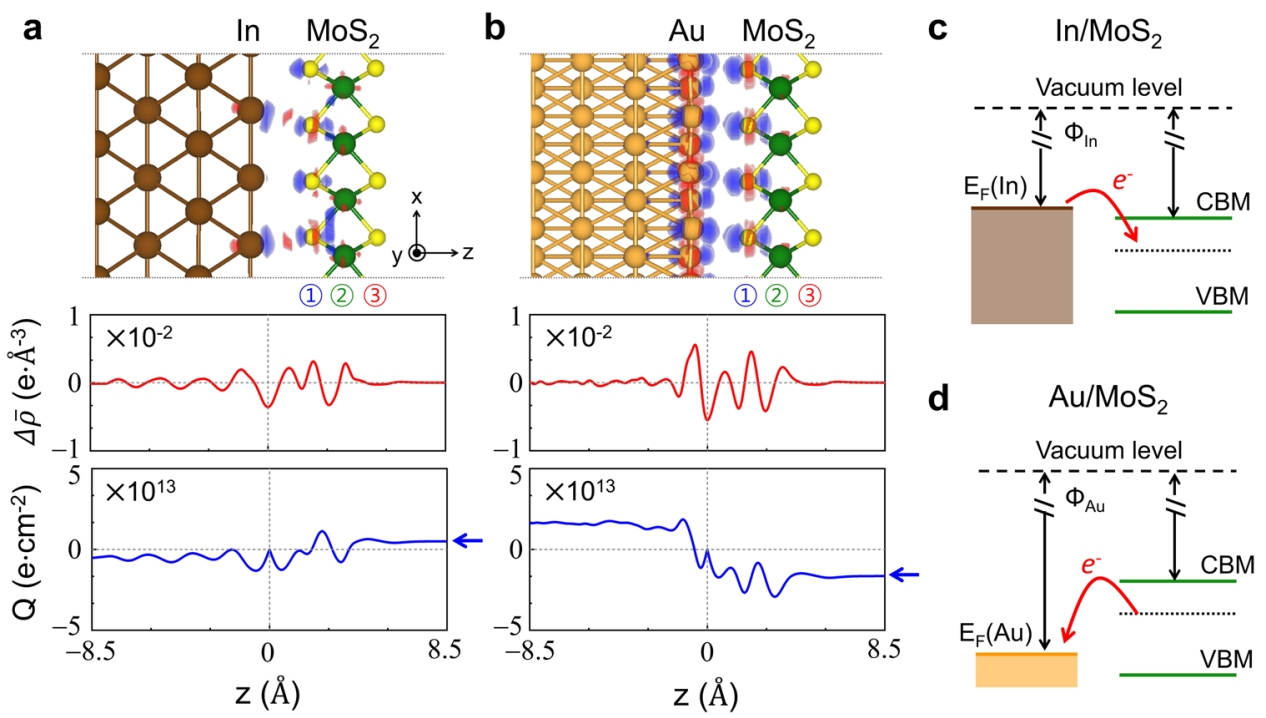

Fig. 3 Charge redistribution properties of the In/MoS interface from DFT calculations. For the a In/MoS 2 and $\mathbf{b}$ Au/MoS interface models, (top panels) DFT-optimized atomic geometries, (middle panels) plane-averaged charge density differences $\Delta \bar{\rho}$, and (bottom panels) total charge transfers $Q$ (bottom) are shown. In the top panels, interfacial charge transfers are overlaid over the atomic structures, with the electron accumulation and depletion regions represented by the red and blue colors, respectively. The isosurface level is $1 \times 10^{-3} e \AA^{-3}$. Schematics of the metal and $\mathrm{MoS}_{2}$ band levels before contact for the $\mathbf{c} \mathrm{In} / \mathrm{MoS}_{2}$ and $\mathbf{d} \mathrm{Au} / \mathrm{MoS}_{2}$ cases. Dotted lines represent the MoS $\mathrm{S}_{2}$ charge neutrality levels, whose energetic positions with respect to the In and Au Fermi levels $E_{\mathrm{F}}$ result in the In-to-MoS ${ }_{2}$ and $\mathrm{MoS}_{2}$-to-Au interfacial charge transfers.

with $\mathrm{MoS}_{2}$. Accordingly, the comparatively smaller In contactinduced structural perturbations consistently seen in our experiment and simulation indicate that In forms even more ideal vdW interactions with $\mathrm{MoS}_{2}$ than Au.

To quantify this conclusion, we calculated the real-space charge density differences $(\Delta \rho)$ at the metal/MoS $\mathrm{S}_{2}$ interfaces according to

$$
\Delta \rho=\rho_{\text {metal } / \operatorname{MoS}_{2}}-\left(\rho_{\mathrm{MoS}_{2}}+\rho_{\text {metal }}\right)
$$

and overlaid the results on the atomic structures in Fig. $3 a, b$ top panels. The plane-averaged $\Delta \bar{\rho}(z)$ for the $\ln / \mathrm{MoS}_{2}$ and $\mathrm{Au} / \mathrm{MoS}_{2}$ contact cases are also presented in Fig. $3 \mathrm{a}$, b middle panels, respectively. A positive (negative) $\Delta \bar{\rho}$ indicates a gain (loss) in electron density, and we find stronger charge redistributions in the $\mathrm{Au} / \mathrm{MoS}_{2}$ contact compared with the In/MoS 2 counterpart. Examining the distribution of $\Delta \bar{\rho}$ between the surface metal layers and the interfacial S layer (1) of $\mathrm{MoS}_{2}$, we further note that due to the "push-back" effect arising from Pauli repulsion there appear charge-depleted (negative $\Delta \bar{\rho}$ ) 2D plane regions close to the metal surfaces (denoted by vertical dotted lines) ${ }^{28,30}$.

Using the minimum $-\Delta \bar{\rho}$ layers as the reference planes $(z=0)$, we calculated along the $\mathrm{MoS}_{2}$ direction the position-dependent 


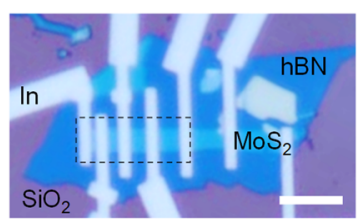

d

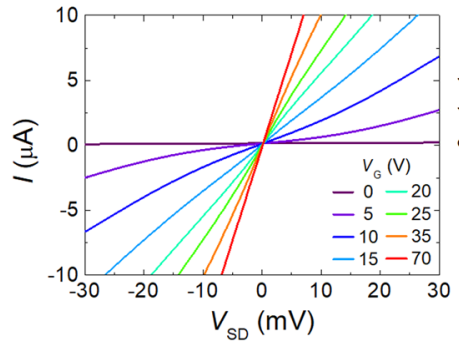

b

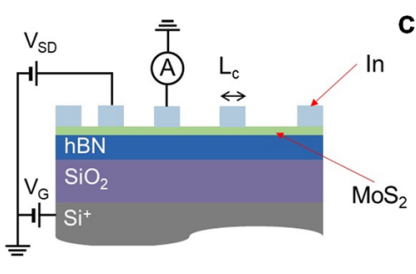

C

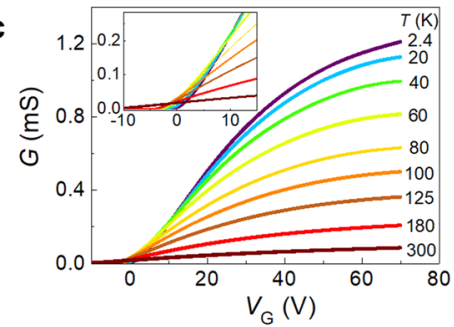

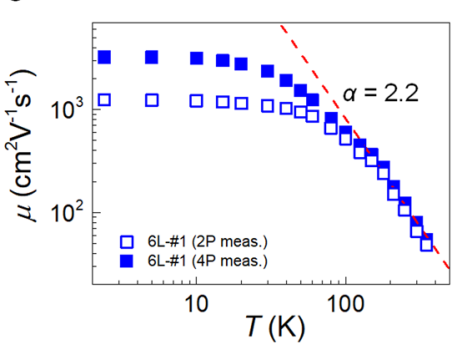

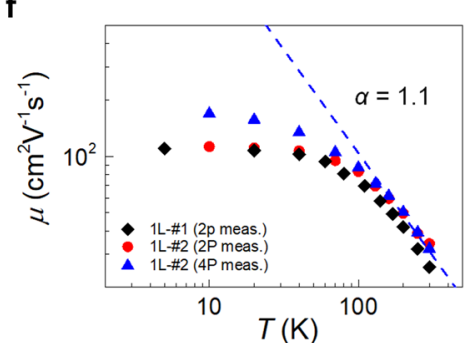

Fig. 4 Basic electrical properties of a few-layer $\mathrm{MoS}_{2}$ field-effect transistor. a Optical image of a $6 \mathrm{~L}-\# 1 \mathrm{MoS}_{2}$ device with multiple In contacts. Scale bar: $5 \mu \mathrm{m}$. b Schematic of $6 \mathrm{~L}-\# 1 \mathrm{MoS}_{2}$ device for the transfer length method (TLM). Here, $L_{c}(=1 \mu \mathrm{m})$ is the length of contact electrode. The measurement scheme shows the two-probe measurement for the $L_{2}=1 \mu \mathrm{m}$ channel. c Conductance $(G)$ as a function of the back-gate voltage $\left(V_{\mathrm{G}}\right)$ at various temperatures. Inset: $G-V_{\mathrm{G}}$ curves near the metal-insulator crossover region. $\mathbf{d} I-V_{\mathrm{SD}}$ Curves for various $V_{\mathrm{G}}$ at $T=2.4 \mathrm{~K}$. e, $\mathbf{f}$ Mobility $(\mu)$ as a function of temperature $(T)$ in log scale for $6 \mathrm{~L}$ and $1 \mathrm{~L}$ devices, respectively. The red and blue dashed lines in e and $\mathbf{f}$ are fitting lines with the relation of $\mu(T) \propto T^{-a}$ with $a=2.2$ and 1.1 for $6 \mathrm{~L}$ and $1 \mathrm{~L}$ devices, respectively.

accumulated interfacial charge transfers according to

$$
Q(z)=\int_{0}^{z} \Delta \bar{\rho}\left(z^{\prime}\right) d z^{\prime}
$$

and displayed the results in Fig. 3a, b bottom panels. Note that $\mathrm{a}$ positive (negative) $Q$ indicates a metal-to- $\mathrm{MoS}_{2}\left(\mathrm{MoS}_{2}\right.$-to-metal) electron transfer upon establishing the metal/MoS 2 interface ${ }^{27}$. We then find that In induces a marginal electron transfer of $+5.0 \times 10^{12}$ electrons $\mathrm{cm}^{-2}$ to $\mathrm{MoS}_{2}$ (left blue arrow in Fig. 3a bottom panel), which is in good quantitative agreement with the above-described estimate of $\sim 1.5 \times 10^{12}$ electrons $\mathrm{cm}^{-2}$ from Raman measurement (see Fig. 2). The marginal electron transfer leads to a weakened FLP, compared to an Au case. For the Au/ $\mathrm{MoS}_{2}$ case, the direction of charge transfer is reversed (electron depletion in $\mathrm{MoS}_{2}$ ), and its magnitude is significantly enhanced to $-1.7 \times 10^{13}$ electrons $\mathrm{cm}^{-2}$ (left blue arrow in Fig. 3b bottom panel). These opposite $\mathrm{MoS}_{2}$ doping characters of In and Au can be understood in terms of the alignment of the $\mathrm{MoS}_{2}$ charge neutrality level $\phi_{1 \mathrm{LMoS}_{2}}^{*} \approx 4.78 \mathrm{eV}$ with respect to the metal work functions $\Phi_{\mathrm{In}} \approx 4.1 \mathrm{eV}$ and $\Phi_{\mathrm{Au}} \approx 5.1 \mathrm{eV}^{31}$, as schematically shown in Fig. 3c, d, respectively (see Supplementary Figs. 5, 6, and 7).

\section{Electrical properties of a $\mathrm{MoS}_{2}$ FET}

Figure $4 a$ and $b$ shows a photograph of a $\mathrm{MoS}_{2}$ FET on a 22-nmthick $h$-BN flake and schematic for the electrical measurement configuration, respectively. The number of $\mathrm{MoS}_{2}$ layers was estimated to be six (6L-\#1 $\mathrm{MoS}_{2}$; see Supplementary Fig. 8). The multiple electrodes for the six-layer (6L) $\mathrm{MoS}_{2}$ flake with different intervals between two neighboring electrodes were designed to measure the contact resistance via the transfer-length method $(T L M)^{13}$, i.e., four FETs with different channel lengths $\left(L_{1}=\right.$ $0.5 \mu \mathrm{m}, L_{2}=1 \mu \mathrm{m}, L_{3}=1.5 \mu \mathrm{m}$, and $L_{4}=2 \mu \mathrm{m}$ from the left channel in the region indicated by a dashed box) with a fixed metal contact length $\left(L_{c}\right)$ as $1 \mu \mathrm{m}$. Here, the widths $(W)$ of all channels were nearly identical at $2 \mu \mathrm{m}$. Figure $4 \mathrm{c}$ shows the twoprobe conductance as a function of the back-gate voltage $\left(G-V_{\mathrm{G}}\right)$ of the $L_{2}$-FET with a source-drain voltage $\left(V_{S D}\right)$ of $30 \mathrm{mV}$ at various temperatures. The conductance decreased for negatively increasing $V_{\mathrm{G}}$ and reached zero near $V_{\mathrm{G}} \approx 0 \mathrm{~V}$ throughout the investigated temperature range, which indicates that the electrical carriers are electrons. The two-probe conductance increased with decreasing $T$ at a given $V_{G}$ for $V_{G}>10 V$, i.e., the device exhibited a metallic behavior. However, the opposite behavior was observed near a depletion region of $0<V_{\mathrm{G}}<10 \mathrm{~V}$ (see the inset of Fig. 4c), representing an insulating character. These behaviors are consistent with the current-voltage $\left(I-V_{\mathrm{SD}}\right)$ curves for various $V_{\mathrm{G}}$ values at $T=2.5 \mathrm{~K}$ in Fig. $4 \mathrm{~d}$. For instance, the $I-V_{\mathrm{SD}}$ curves for $V_{\mathrm{G}}>10 \mathrm{~V}$ and $0<V_{\mathrm{G}}<10 \mathrm{~V}$ show linear and nonlinear characteristics, respectively. We consider that the linearity and non-linearity originate from the field and thermionic emission through a Schottky barrier, respectively. The fourprobe measurements for the $L_{2}$ channel also showed a similar $V_{\mathrm{G}}$ value for the metal-insulator crossover location (see Supplementary Fig. 9). This result indicates that the transport in the $\mathrm{MoS}_{2}$ channel near the conduction-band edge also contributes to the crossover behavior, as well as the Schottky barrier. We also extracted the on/off ratio of $\sim 10^{5}$ and $10^{3}-10^{5}$ of other monolayer (1L-\#1) and $6 \mathrm{~L}(6 \mathrm{~L}-\# 2)$ devices, respectively, as shown in Supplementary section 5 . The ratios satisfy a condition for logic gate applications. On the other hand, the on-current densities of $1 \mathrm{~L}-\# 1 \mathrm{MoS}_{2}$ and $6 \mathrm{~L}-\# 2 \mathrm{MoS}_{2}$ devices reached $\sim 60 \mu \mathrm{A}$ $\mu \mathrm{m}^{-1}$ and $\sim 200 \mu \mathrm{A \mu m} \mathrm{m}^{-1}$, respectively. The $6 \mathrm{~L}-\# 2 \mathrm{MoS}_{2}$ device also showed a stability against aging at least for 40 days for $-1 \mathrm{~V}$ $<V_{\mathrm{SD}}<1 \mathrm{~V}$ (see Supplementary Fig. 11d).

In Fig. $4 \mathrm{e}$, we show the field-effect mobility $(\mu)$ of the $6 \mathrm{~L}-\# 1$ $\mathrm{MoS}_{2}$ device as a function of $T$ obtained from the two-probe (open squares) and four-probe (closed squares) measurement schemes. The mobility was obtained at the local maximum location in the $\mu-V_{\mathrm{G}}$ curves (see Supplementary Fig. 12). For $T>100 \mathrm{~K}$, in both cases, the data were fitted with a relation of $\mu(T)=\mu_{0} T^{-a}$ with $a=2.2$ as shown by the dashed red line. This value is close to the expected value for bulk $\operatorname{MoS}_{2}(a=2.6)$ with the optical phonon scattering as a dominant scattering mechanism ${ }^{12,32,33}$. At room $T$, $\mu \sim 50 \mathrm{~cm}^{2} \mathrm{~V}^{-1} \mathrm{~s}^{-1}$ values were obtained for both cases. However, the two-probe and four-probe $\mu$ values were saturated with decreasing temperature in the region $T<20 \mathrm{~K}$ at 1200 and $3200 \mathrm{~cm}^{2} \mathrm{~V}^{-1} \mathrm{~s}^{-1}$, respectively. The saturation behavior in low- $T$ regions is known to occur when the impurity scattering assumes a dominant role while the phonon-scattering effect is suppressed ${ }^{34}$. By contrast, the mobility for two different $1 \mathrm{~L} \mathrm{MoS} 2$ devices (1L-\#1 and 1L-\#2; see Supplementary Figs. 10c, 13, respectively) displayed in Fig. $4 \mathrm{f}$ show a $T$ dependence with $a=1.1$ for $T>100 \mathrm{~K}$, which is 

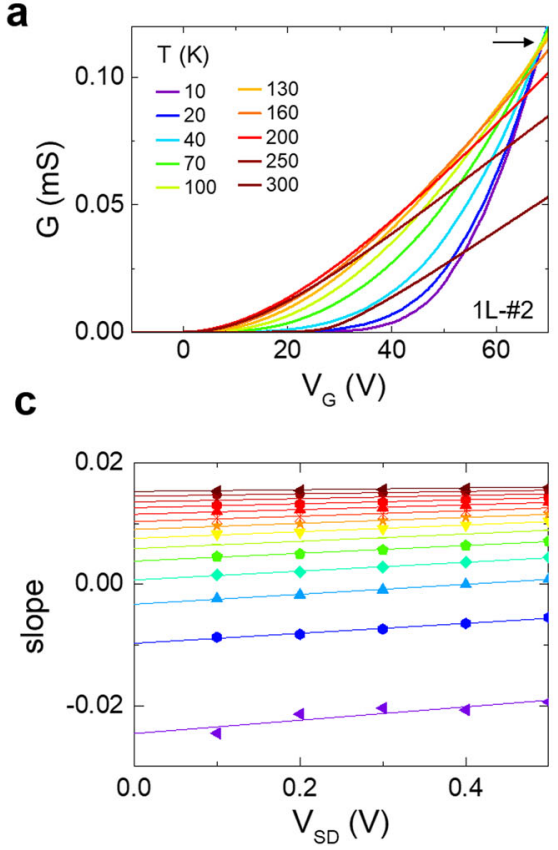

b
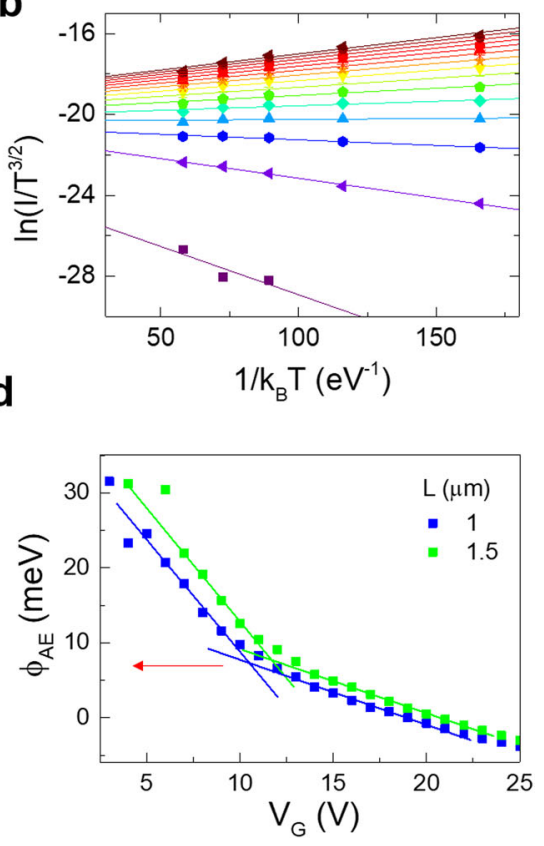

Fig. 5 Schottky barrier height at $\operatorname{In} / \mathrm{MoS}_{2}$ contacts. a $G-V_{\mathrm{G}}$ curves at various temperatures obtained from the $L=1 \mu \mathrm{m}$ channel of $1 \mathrm{~L}-\# 2$ $\mathrm{MoS}_{2}$ device, indicated by a dashed box in Supplementary Fig. 13a. b Scattered points: $\ln \left(I / T^{3 / 2}\right)$ as a function of $1 / k_{\mathrm{B}} T$ with $V_{\mathrm{SD}}=0.5 \mathrm{~V}$ from $V_{\mathrm{G}}=0 \mathrm{~V}$ to $70 \mathrm{~V}$ with $5 \mathrm{~V}$ spacing (from bottom to top) obtained from the $L=1 \mu \mathrm{m}$ channel. Solid lines: fitting result to obtain slope values for each $V_{G}$ value. c Scattered points: slope as a function of $V_{S D}$ for various $V_{G}(5 \mathrm{~V}$ to $70 \mathrm{~V}$ with $5 \mathrm{~V}$ spacing from bottom to top). Solid lines: fitting result to obtain $\phi_{\mathrm{AE}}$ at $V_{\mathrm{SD}}=0 \mathrm{~V}$ for each $V_{\mathrm{G}}$ value. $\mathbf{d} \phi_{\mathrm{AE}}$ as a function of $V_{\mathrm{G}}$ for $L=1$ and $1.5 \mu \mathrm{m}$ channels. The arrow indicates the $\phi_{\mathrm{SB}}(\sim 7 \mathrm{meV})$ for the $1 \mathrm{~L}-\# 2 \mathrm{MoS}_{2}$ device.

close to the prediction of the acoustic phonon scattering becoming a dominant scattering mechanism for a monolayer $\mathrm{MoS}_{2}$ (ref. ${ }^{33}$ ).

For comparison, we also fabricated $\mathrm{MoS}_{2}$ devices with In contacts covered with $\mathrm{Au}$, where the sample holder was a copper block prepared without a liquid-nitrogen cooling process. In this case, we found that the reproducibility was not high enough to obtain the consistent Ohmic-like behavior in the $I-V_{S D}$ curves, confirming the critical role of our substrate cooling technique (see Supplementary Fig. 14).

Schottky barrier height at In/MoS 2 contacts

We evaluated $\phi_{\mathrm{SB}}$ at the $\mathrm{In} / \mathrm{MoS}_{2}$ interface because this parameter plays a critical role in determining the contact resistance between a metal and a semiconductor ${ }^{4}$. For this purpose, it is necessary to measure the activation energy $\left(\phi_{\mathrm{AE}}\right)$ at the contacts in the thermionic emission region 6 . Here, because the $V_{G}$ range for the insulating region is larger than that of the $6 \mathrm{~L}-\# 1 \mathrm{MoS}_{2}$ device (see Fig. 4c), we used the $1 \mathrm{~L}-\# 2 \mathrm{MoS}_{2}$ device with the $L=1 \mu \mathrm{m}$ channel shown in Supplementary Fig. 13a. Figure 5a shows the resulting $G-V_{G}$ curves for various temperatures obtained by the twoprobe measurement. In this case, the crossover $V_{\mathrm{G}}$ between the insulating and metallic regions was located at a relatively higher $V_{G}$ ( $65 \mathrm{~V})$ than that of the $6 \mathrm{~L}-\# 1 \mathrm{MoS}_{2}$ device, as indicated by an arrow. The $G$ values increased with increasing $T$ for $T<130 \mathrm{~K}$ at $V_{\mathrm{G}}<65 \mathrm{~V}$ and decreased for $T>200 \mathrm{~K}$ in the examined $V_{\mathrm{G}}$ range. When the thermionic emission is dominant, the current crossing a metal/2D system is described by the relation

$I_{\mathrm{d}}=A^{*} T^{3 / 2} \exp \left(\frac{-e \phi_{\mathrm{AE}}}{k_{\mathrm{B}} T}\right)\left[\exp \left(\frac{e V_{\mathrm{SD}}}{\eta k_{\mathrm{B}} T}\right)-1\right]$,

where $A^{*}$ is the Richardson constant, $e$ is the elementary charge, $k_{\mathrm{B}}$ is the Boltzmann constant, and $\eta$ is the ideality factor that accounts for a lower barrier height due to image charging ${ }^{12}$. Figure $5 \mathrm{~b}$ shows $\ln \left(I / T^{3 / 2}\right)$ as a function of $1 / k_{\mathrm{B}} T$ at $V_{\mathrm{SD}}=0.5 \mathrm{~V}$ for various $V_{\mathrm{G}}$ values from $0 \mathrm{~V}$ to $70 \mathrm{~V}$ with $5 \mathrm{~V}$ spacing (from bottom to top). The slopes of $\ln \left(I / T^{3 / 2}\right)-1 / k_{\mathrm{B}} T$ curves are related to $\phi_{\mathrm{AE}}$ as $\phi_{\mathrm{AE}}=V_{\mathrm{SD}} / \eta$ - slope. After obtaining the slopes corresponding to representative $V_{S D}$ values, we plotted the slope as a function of $V_{\mathrm{SD}}$ for various $V_{\mathrm{G}}(5 \mathrm{~V}$ to $70 \mathrm{~V}$ with $5 \mathrm{~V}$ spacing from bottom to top) to obtain $\phi_{\mathrm{AE}}$ at $V_{\mathrm{SD}}=$ $0 \mathrm{~V}$, as shown in Fig. $5 \mathrm{c}$. Finally, we plotted $\phi_{\mathrm{AE}}$ vs. $V_{\mathrm{G}}$ to obtain $\phi_{\mathrm{SB}}$ as blue squares in Fig. $5 \mathrm{~d}$. Near the depletion region, $\phi_{\mathrm{AE}}$ is linearly lowered with increasing $V_{\mathrm{G}}$ when the thermally activated transport is dominant and changes its slope when the field-emission transport is accounted at the Schottky barrier. Thus, the crossover point between them occurs when the band flattens, where, the value of $\phi_{\mathrm{AE}}$ becomes equal to the value of $\phi_{\mathrm{SB}}{ }^{6}$. To find $V_{\mathrm{G}}$ making the band flat, we plotted two linear blue lines on the blue squares in Fig. $5 \mathrm{~d}$. The two curves meet at $V_{\mathrm{G}} \approx 10 \mathrm{~V}$, where the flat band is believed to form, and we estimated $\phi_{\mathrm{SB}} \approx 7 \mathrm{meV}$ at the corresponding $\phi_{\mathrm{AE}}$ for the $\ln / \operatorname{MoS}_{2}(n=1)$ contact. In addition, we also obtained a similar value for the $L=1.5 \mu \mathrm{m}$ channel as indicated by green squares and green fit lines in Fig. $5 d$ (see also Supplementary Fig. 13a). This value is in a similar range obtained from a $\mathrm{Co} / h-\mathrm{BN}$ contact with a monolayer $\mathrm{MoS}_{2}$ (ref. ${ }^{10}$ ). Such a low $\phi_{\mathrm{SB}}$ at the $\mathrm{In} / \mathrm{MoS}_{2}$ contact could allow the field-emission to play a dominant role for the transport across the $\mathrm{In} / \mathrm{MoS}_{2}$ contact.

\section{Contact resistance at $\mathrm{In} / \mathrm{MoS}_{2}$ contacts}

On the basis of the TLM measurements (see Supplementary Fig. 15) with multiple channels (see Fig. 4a), we extracted the contact resistance $\left(R_{c} W\right)$ as a function of $n_{\mathrm{e}}$ of the $6 \mathrm{~L}-\# 1 \mathrm{MoS}_{2}$ device at representative temperatures; the results are shown in Fig. $6 \mathrm{a}$ (solid squares; see also Supplementary Table 2). Here, $n_{\mathrm{e}}$ was estimated from the relation $n_{\mathrm{e}}=\left(e \mu R_{\mathrm{sh}}\right)^{-1}$. We note that, for the consistency, the sheet resistance $R_{\mathrm{sh}}$ as well as mobility $\mu$ were obtained from the four-probe data, although $R_{\text {sh }}$ could have been extracted from the TLM method. The obtained contact resistance includes serial resistances of In and Ti/Au electrodes (see supplementary Fig. 16). At a given $T, R_{c} W$ decreased with increasing $n_{\mathrm{e}}$. The contact 
a

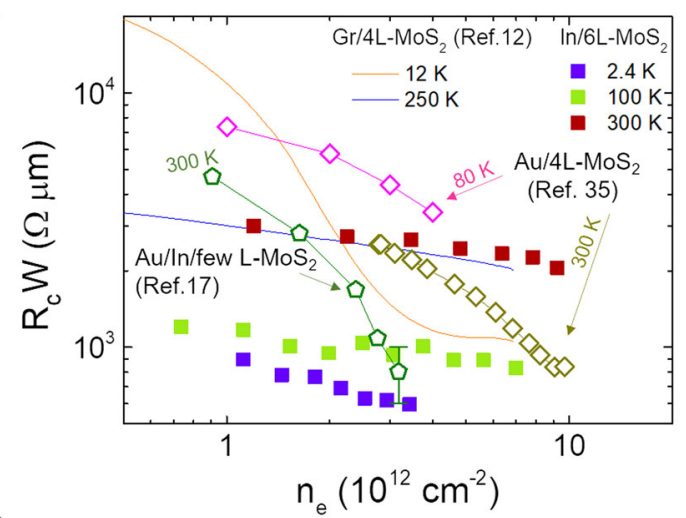

b

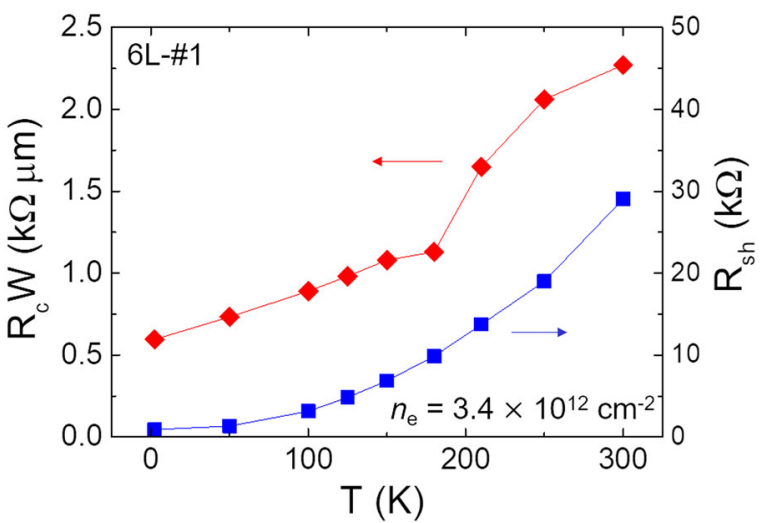

Fig. 6 Contact resistance with carrier density at In/MoS contacts. a Contact resistance $\left(R_{\mathrm{c}} W\right)$ as a function of carrier density $\left(n_{\mathrm{e}}\right)$ of $6 \mathrm{~L}-$ $\# 1 \mathrm{MoS}_{2}$ device at various temperatures (scattered solid squares) with other works (solid curves: graphene $(\mathrm{Gr}) / 4 \mathrm{~L}-\mathrm{MoS}_{2}$, opened diamonds: $\mathrm{Au} / 4 \mathrm{~L}-\mathrm{MoS}_{2}$, opened pentagons: Au/ln/few L-MoS 2 ). b $R_{\mathrm{c}} W$ and sheet resistance $\left(R_{\mathrm{sh}}\right)$ of $6 \mathrm{~L}-\# 1 \mathrm{MoS}_{2}$ device as a function of $T$ at $n_{\mathrm{e}}=3.4 \times 10^{12} \mathrm{~cm}^{-2}$.

resistance is given by ${ }^{4,5}$

$$
R_{\mathrm{c}} W\left(n_{\mathrm{e}}, T\right)=\sqrt{R_{\mathrm{sh}}\left(n_{\mathrm{e}}, T\right) \rho_{\mathrm{c}}\left(n_{\mathrm{e}}, T\right)}
$$

which is only valid for $L_{c} \gg L_{T}$. Here, $\rho_{c}$ is the specific contact resistivity and $L_{T}\left(=\sqrt{\rho_{\mathrm{c}} / R_{\mathrm{sh}}}\right)$ is the transfer length, which represents the average distance that charge carriers flow in a semiconductor beneath the contact before they completely transport to the electrode. Supplementary Fig. $15 \mathrm{C}$ shows that our device satisfied this condition with $L_{c} \approx 1 \mu \mathrm{m}$ and $L_{T} \approx 0.1 \mu \mathrm{m}$. Equation (4) implies that $R_{\mathrm{c}} W$ decreases with increasing $n_{\mathrm{e}}$ because both $R_{\mathrm{sh}}$ and $\rho_{\mathrm{c}}$ generally decrease with increasing $n_{\mathrm{e}}$. At a fixed $n_{\mathrm{e}}$ the thermionic emission charge transport mechanism across the Schottky barrier predicts that $R_{\mathrm{c}} W$ will increase with decreasing $T$ because the thermionic emission will be suppressed with lowering $T^{35}$. On the other hand, $R_{c} W$ in our measurements decreased with decreasing $T$, as shown by scattered red diamonds in Fig. $6 \mathrm{~b}$ for the case measured at $n_{\mathrm{e}}=3.4 \times 10^{12} \mathrm{~cm}^{-2}$. The contact resistance decreased from 2.3 to $0.6 \mathrm{k} \Omega \mu \mathrm{m}$ when $T$ was decreased from room temperature to $2.4 \mathrm{~K}$. This behavior, which has been reported in several previous works such as graphene/ $\mathrm{MoS}_{2}$ (ref. ${ }^{12}$ ) and $\mathrm{Pd} / \mathrm{graphene}$ contacts ${ }^{36}$, is considered as an evidence for the non-dominant role of thermionic emission for the transport across a contact. Scattered squares in Fig. $6 \mathrm{~b}$ also show $R_{\text {sh }}$ as a function of $T$ at $n_{\mathrm{e}}=3.4 \times 10^{12} \mathrm{~cm}^{-2}$, where $R_{\text {sh }}$ decreased with decreasing $T$ because the phonon scattering is reduced.
Sheet resistance vs. specific contact resistivity

We further analyzed the mechanism of charge transport across In/ $\mathrm{MoS}_{2}$ contacts with experimental data in detail. We first examined which component between $R_{\mathrm{sh}}$ and $\rho_{\mathrm{c}}$ predominantly determines the contact resistance of the In/MoS 2 contact. For comparison, we included in Fig. 6a other values reported in the literature; graphene(Gr)/four-layer (4L)-MoS contact $^{12}, \mathrm{Au} / 4 \mathrm{~L}-\mathrm{MoS}_{2}$ (ref. ${ }^{35}$ ) and $\mathrm{Au} / \mathrm{In} / \mathrm{few}$-layer $\mathrm{MoS}_{2}\left(\right.$ ref. ${ }^{17}$ ). In the case of Gr/4L-MoS 2 , the graphene functions as a work-function-controllable contact material, which leads to a lower contact resistance, i.e., $R_{c} W \approx$ $1 \mathrm{k} \Omega \mu \mathrm{m}$ at $n_{\mathrm{e}}>4 \times 10^{12} \mathrm{~cm}^{-2}$ and $T=12 \mathrm{~K}$ (see the red curve in Fig. 6a). Although both the Gr-and In-contact $\mathrm{MoS}_{2}$ devices gave a similar minimum $R_{c} W$ at cryogenic temperatures, we conclude that the transport mechanisms at the contacts rather differ from each other. For the In/MoS contact case, $R_{c} W$ decreased with decreasing $T$ in the examined $n_{\mathrm{e}}$ range, representing the field emission (or tunneling) for all examined $T$ and $n_{\mathrm{e}}$ ranges. However, the $R_{\mathrm{c}} W-n_{\mathrm{e}}$ curves obtained at $T=12$ and $250 \mathrm{~K}$ for the $\mathrm{Gr} / 4 \mathrm{~L}-$ $\mathrm{MoS}_{2}$ device suggest that the left and right sides with respect to $n_{\mathrm{e}} \approx 2 \times 10^{12} \mathrm{~cm}^{-2}$ followed the thermionic and field emissions at the contact, respectively. At $T=12 \mathrm{~K}$ for the $\mathrm{Gr} / 4 \mathrm{~L}-\mathrm{MoS}_{2}$ device, although the $R_{\mathrm{c}} W$ of $\sim 1 \mathrm{k} \Omega \mu \mathrm{m}$ was relatively insensitive to the variation of $n_{\mathrm{e}}$ in the range from $4 \times 10^{12}$ to $7 \times 10^{12} \mathrm{~cm}^{-2}$, it rapidly changed from $1 \mathrm{k} \Omega \mu \mathrm{m}$ to $6 \mathrm{k} \Omega \mu \mathrm{m}$ when $n_{\mathrm{e}}$ decreased from $3 \times 10^{12} \mathrm{~cm}^{-2}$ to $1.5 \times 10^{12} \mathrm{~cm}^{-2}$. In the case of the $\mathrm{Au} / 4 \mathrm{~L}-$ $\mathrm{MoS}_{2}$ contact, on the other hand, $R_{c} W$ was increased with decreasing $T$ representing the thermionic emission for $n_{\mathrm{e}}<4 \times$ $10^{12} \mathrm{~cm}^{-2}$. While the Au/ln/few layer-MoS 2 contact provides a relatively low- $R_{\mathrm{c}} W$ level for $n_{\mathrm{e}}>1.5 \times 10^{12} \mathrm{~cm}^{-2}$ at $T=300 \mathrm{~K}$, it is hard to judge the transport mechanism for the In contact because of the absence of the $T$-dependence of $R_{\mathrm{C}} W$ in the experiment. Then, for the In/6L-\#1 $M_{0} S_{2}$ device, the $R_{\mathrm{C}} W$ was lowered with decreasing $T$ at a given $n_{\mathrm{e}}$ for $1 \times 10^{12} \mathrm{~cm}^{-2}<n_{\mathrm{e}}<1 \times 10^{13} \mathrm{~cm}^{-2}$, representing an Ohmic-like behavior based on the field-emission mechanism in the examined $n_{\mathrm{e}}$ region. In our In/6L-MoS ${ }_{2}$ device (see Fig. 7a), $R_{\text {sh }}$ varies in the range from 1 to $80 \mathrm{k} \Omega$ when $\rho_{\mathrm{c}}$ only varies from $5 \times 10^{-6}$ to $5 \times 10^{-7} \Omega \mathrm{cm}^{2}$, as shown by two dashed lines, for $R_{c} W$ changing from 0.6 to $\sim 3 \mathrm{k} \Omega \mu \mathrm{m}$. This result indicates that $R_{\mathrm{sh}}$ plays a dominant role in determining $R_{\mathrm{c}} W$ in the fieldemission region.

We also obtained $R_{\mathrm{c}} W$ from the $1 \mathrm{~L}-\# 2 \mathrm{MoS}_{2}$ device on a 40-nmthick $h$-BN flake (see Fig. 7b and Supplementary Fig. 13 for the thickness profile). In Fig. $7 \mathrm{~b}$, the $R_{\mathrm{c}} W$ values were extracted via the TLM with three channels $(L=0.5,1,1.5 \mu \mathrm{m})$ as shown in Supplementary Fig. $13 a$. For three $V_{G-t h}$ conditions of 35,40 , and $45 \mathrm{~V}, R_{\mathrm{c}} W$ decreased with decreasing $T$ in the range $250 \geq T \geq$ $100 \mathrm{~K}$, representing the field emission. Here, $V_{\mathrm{G}-\mathrm{th}}=V_{\mathrm{G}}-V_{\mathrm{th}}$ and $V_{\mathrm{th}}$ is a threshold voltage. At $V_{\mathrm{G}-\mathrm{th}}=45 \mathrm{~V}, R_{\mathrm{c}} W$ reached $\sim 1 \mathrm{k} \Omega \mu \mathrm{m}$ at $T=100 \mathrm{~K}$ as the minimum value obtained from the $1 \mathrm{~L}-\mathrm{MoS}_{2}$ device. Although this value is similar to that obtained from the $6 \mathrm{~L}-$ $\mathrm{MoS}_{2}$ device at a similar $T$ range (see Fig. 6a), the contact resistance for the monolayer $\mathrm{MoS}_{2}$ is higher than that of multilayer $\mathrm{MoS}_{2}$. The reason could be related to the relatively low affinity energy in the monolayer $\mathrm{MoS}_{2}$. Interestingly, $R_{c} W$ increased with decreasing $T$ for $T<100 \mathrm{~K}$ under all $V_{\mathrm{G}-\mathrm{th}}$ conditions. In this region, the $\mathrm{MoS}_{2}$ channel also exhibited an insulating behavior in $G-V_{\mathrm{G}}$ curves for various temperatures of Fig. $5 \mathrm{a}$ for $V_{\mathrm{G}}<60 \mathrm{~V}$ and $T<$ $100 \mathrm{~K}$. Thus, it indicates that the increase of $R_{\mathrm{sh}}$ with decreasing $T$ in the insulating phase plays a dominant role in determining the contact resistance at $T<100 \mathrm{~K}$. We note that this non-Ohmic behavior could not be improved by the contact engineering because the behavior originates from the intrinsic property of $\mathrm{MoS}_{2}$ itself. This implies that the manipulation of the metalinsulator crossover gate voltage could be crucial to get a better contact property in a mono-layer $\mathrm{MoS}_{2}$ device. In Fig. 7a (see also Supplementary Table 2), we compared the lowest achievable contact resistance as a function of $R_{\mathrm{sh}}$ from previous reports with 
a

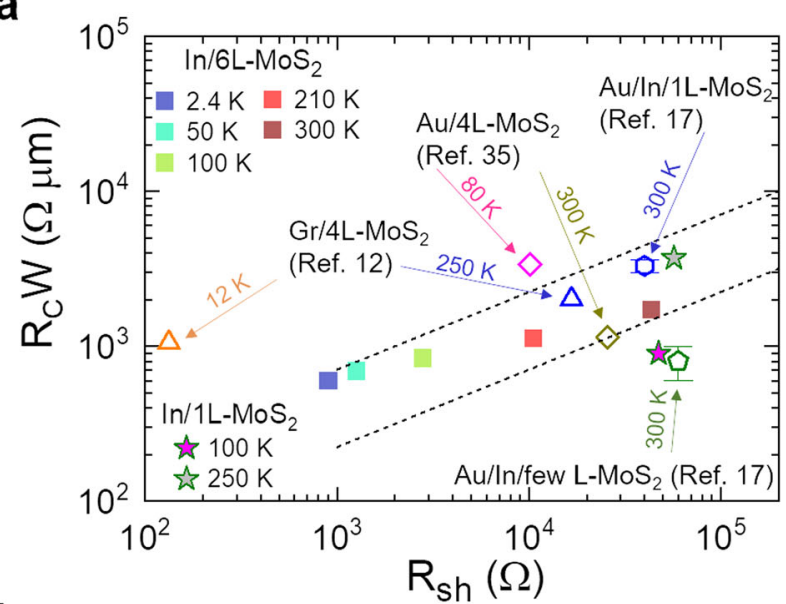

b

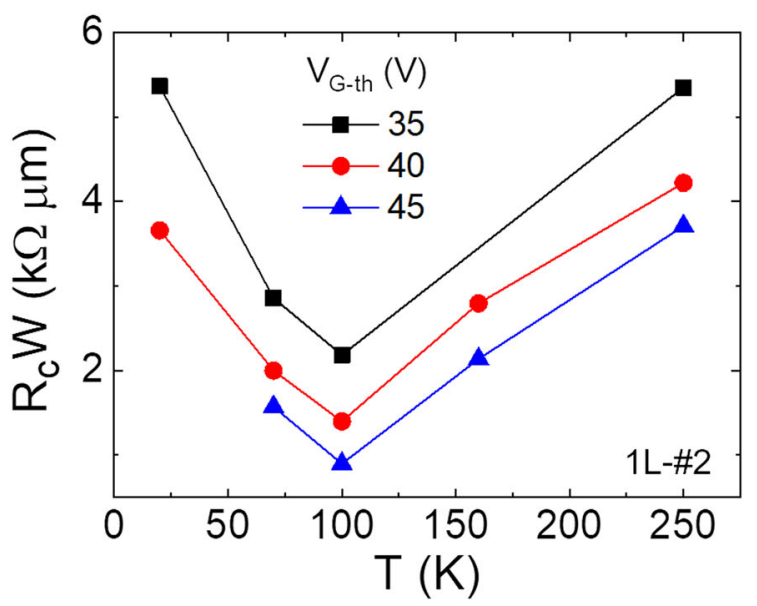

Fig. 7 Contact resistance with sheet resistance at $\ln / \mathrm{MoS}_{2}$ contacts. a Minimum $R_{c} W$ as a function $R_{\text {sh }}$ of $6 \mathrm{~L}-\# 1$ and $1 \mathrm{~L}-\# 2$ $\mathrm{MoS}_{2}$ devices at various temperatures (scattered solid squares and stars, respectively) with previous works (opened triangles: graphene (Gr)/4L-MoS 2 , opened diamonds: Au/4L-MoS 2 , opened pentagons: $\mathrm{Au} / \mathrm{In} / \mathrm{few}$ (1) L-MoS ${ }_{2}$ ). The upper and lower dashed lines were obtained with $\rho_{\mathrm{c}}=5 \times 10^{-6} \Omega \mathrm{cm}^{2}$ and $5 \times 10^{-7} \Omega \mathrm{cm}^{2}$, respectively. b $R_{\mathrm{c}} W-T$ curves for various $V_{\mathrm{G}-\mathrm{th}}$ conditions of $1 \mathrm{~L}-\# 2 \mathrm{MoS}_{2}$ device.

ours. For the In/MoS 2 device, the contact resistance decreased with decreasing $R_{\mathrm{sh}}$ and reached $\sim 0.6 \mathrm{k} \Omega \mu \mathrm{m}$ as the lowest value at $R_{\text {sh }} \sim 1 \mathrm{k} \Omega$ and $T=2.4 \mathrm{~K}$. At $T=300 \mathrm{~K}$, on the other hand, the Au/ $\mathrm{In} / \mathrm{few}$ layer-MoS 2 device showed the lowest value of $\sim 0.6 \mathrm{k} \Omega \mu \mathrm{m}$ at $R_{\mathrm{sh}} \sim 60 \mathrm{k} \Omega$. In addition, for monolayer cases, our $\mathrm{In} / 1 \mathrm{~L}-\# 2 \mathrm{MoS}_{2}$ contact provided $0.9-4 \mathrm{k} \Omega \mu \mathrm{m}$ at $R_{\mathrm{sh}} \sim 50 \mathrm{k} \Omega$, which are comparable to those obtained from the Au/In/1L-MoS 2 contact that showed $\sim 3 \mathrm{k} \Omega \mu \mathrm{m}$ at $R_{\mathrm{sh}} \sim 40 \mathrm{k} \Omega$.

Atomistic origins of the field emission-dominated charge transport across In/MoS 2 contacts

Analyzing the electronic structures of the $\operatorname{In} / \mathrm{MoS}_{2}$ and $\mathrm{Au} / \mathrm{MoS}_{2}$ interfaces obtained from DFT calculations, we finally identify the atomistic mechanisms of the experimentally observed Ohmiclike charge transport behavior. It should be noted that, while there exist in the literature several theoretical studies that examined the Schottky barriers in metal/TMDC interfaces ${ }^{30,37-40}$, the In/TMDC case has been rarely treated ${ }^{41}$. We show the calculated band structures at the In/MoS 2 and Au/MoS 2 contacts in Fig. 8a, b, respectively, and particularly display the projected bands of Mo-4d (green circles in Fig. 8a, b), In-5 $p_{z}$ (wine filled circles in Fig. 8a), and Au-6s (orange circles in Fig. 8b) orbitals. From the band structures, one could determine the electron $\phi_{\mathrm{SB}}$ by measuring the energy level difference between the conduction band minimum (CBM) edge (upper solid purple line) and the Fermi level $E_{\mathrm{F}}$ (dashed purple line) of metal/MoS ${ }_{2}$ contacts. However, the comparison of the two bands indicate that identifying the electron $\phi_{\mathrm{SB}}$ in the In/ $\mathrm{MoS}_{2}$ contact is a non-trivial matter due to the strong density of in-gap states appearing below and around the $\mathrm{MoS}_{2}$ CBM region (green circles between CBM and $E_{F}$ in Fig. 8a; note the absence of such in-gap states in the Au/MoS2 contact in Fig. 8b). To circumvent this difficulty, we first extracted the hole $\phi_{\mathrm{SB}}$ from the In/MoS ${ }_{2}$ junction band structure (see also Supplementary Fig. 5). Next, calculating the band structure of the pristine monolayer $\mathrm{MoS}_{2}$ using the simulation cell of the corresponding In/MoS junction model (Supplementary Fig. 6), we determined the electron $\phi_{\mathrm{SB}}$ by subtracting the hole $\phi_{\mathrm{SB}}$ from the calculated band gap of monolayer $\mathrm{MoS}_{2}(1.94 \mathrm{eV}$ ) (Supplementary Fig. 7). The obtained electron $\phi_{\mathrm{SB}}$ values were $0.31 \mathrm{eV}$ and $0.72 \mathrm{eV}$ for the $\mathrm{In} /$ $\mathrm{MoS}_{2}$ and $\mathrm{Au} / \mathrm{MoS}_{2}$ junctions, respectively. The In/MoS 2 electron, $\phi_{\mathrm{SB}}$ value of $0.31 \mathrm{eV}$ is comparable to a previous estimate of $0.47 \mathrm{eV}^{41}$ and much larger than the experimentally identified marginal $\phi_{\mathrm{SB}}$ values. We thus conclude that the experimentally observed $\phi_{\mathrm{SB}}$ is an effective $\phi_{\mathrm{SB}}$ that has been significantly reduced from the intrinsic $\phi_{S \mathrm{SB}}$ by strong density of in-gap states.

Having been identified as the distinguishing feature, the large in-gap states arising in the In/MoS 2 contact should be the source of the marginal effective $\phi_{\mathrm{SB}}$ and Ohmic-like transport and deserve further attention. Disregarding this factor, for example, can lead to the conclusion that Au would form a better contact than In for $\mathrm{MoS}_{2}{ }^{41}$, but the in-gap states of the In/MoS 2 contact remains unexplored ${ }^{37,38}$. To examine the nature of in-gap states in the In/MoS interface in comparison with that of the Au/MoS counterpart, we show in Fig. $8 c$, d how the In-5 $p_{z}$ (pink filled line) and $\mathrm{Au}-6 \mathrm{~s}$ (orange filled line) projected density of states (PDOS) evolve into the metal-contacting (1) S-3p layer, (2) Mo- $4 d$ layer, and (3) the outer S-3p layer PDOS of $\mathrm{MoS}_{2}$ for the In/MoS 2 and Au/MoS contacts, respectively. It is immediately notable that, while the Au-6s PDOS are uniformly distributed across the $\mathrm{MoS}_{2}$ bandgap (Fig. 8d first panel), the $\ln -5 p_{z}$ PDOS show energetically very asymmetric distribution such that they start from a negligible level near the $\mathrm{MoS}_{2}$ VBM region and increase significantly toward the $\mathrm{MoS}_{2}$ CBM region (Fig. 8c first panel). The MoS VBM and CBM PDOS are mostly composed of Mo-4d orbitals (compare the second/fourth panels with the second panels in Fig. 8c, d). Then, we find for the In/MoS contact case that very strong density of ingap states are formed between $E_{\mathrm{F}}$ and $\mathrm{MoS}_{2}$ CBM position (Fig. 8c third panel). As schematically depicted by the thick arrow in Fig. 8c, electrons injecting from In into these $\mathrm{MoS}_{2}$ in-gap states then should result in the experimentally observed field-emissiontype charge transport accompanied by a negligible effective $\phi_{\mathrm{SB}}$. On the other hand, the corresponding Mo-4d PDOS around $E_{\mathrm{F}}$ for the Au contact case are relatively marginal, making such a charge injection mechanism ineffective (schematically represented by a thin arrow in Fig. 8d). In Fig. 8e, f, we additionally visualized in real space the $E_{\mathrm{F}}$-region $\mathrm{In}-\mathrm{MoS}_{2}$ and $\mathrm{Au}-\mathrm{MoS}_{2}$ hybridized DOS, respectively. The stronger hybridization between In and $\mathrm{MoS}_{2}$ states than that between $\mathrm{Au}$ and $\mathrm{MoS}_{2}$ states then provides an intuitive explanation how the Ohmic-like charge transport is achieved for the In/MoS 2 interface in spite of the very small interfacial charge transfer (see Fig. 3).

In summary, carrying out a combined experimental and theoretical investigation for an ultraclean vdW contact between an elemental metal In (without alloying) and semiconductor $\mathrm{MoS}_{2}$, we revealed the mechanism of Ohmic charge transport across the In/MoS 2 vdW interface. For the single- and few-layer $\mathrm{MoS}_{2}$ devices, the contact resistance decreased with decreasing temperatures for $100 \leq T \leq 300 \mathrm{~K}$, indicating the field-emission mechanism for the Ohmic-like contact transport. The contact resistance was sensitive to the change of sheet resistance of $\mathrm{MoS}_{2}$, rather than 


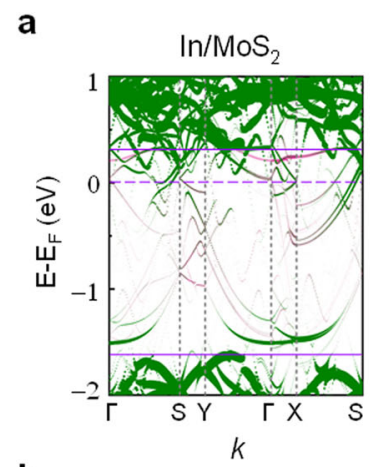

b

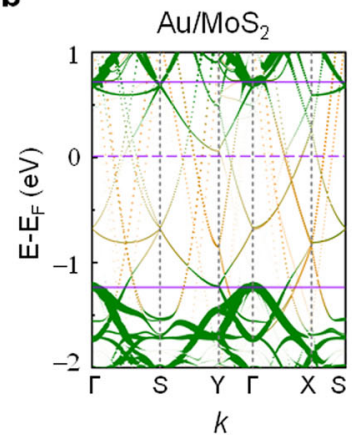

c

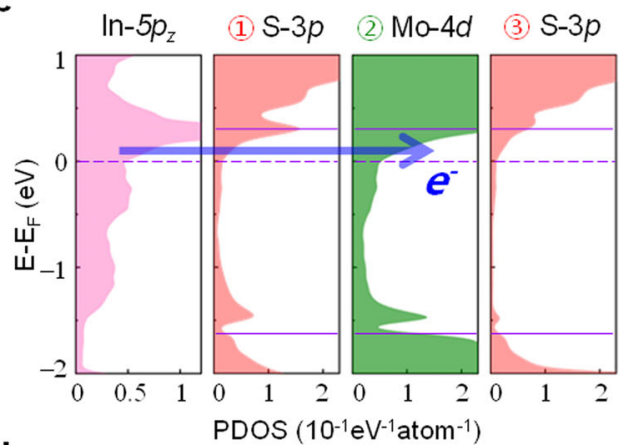

d

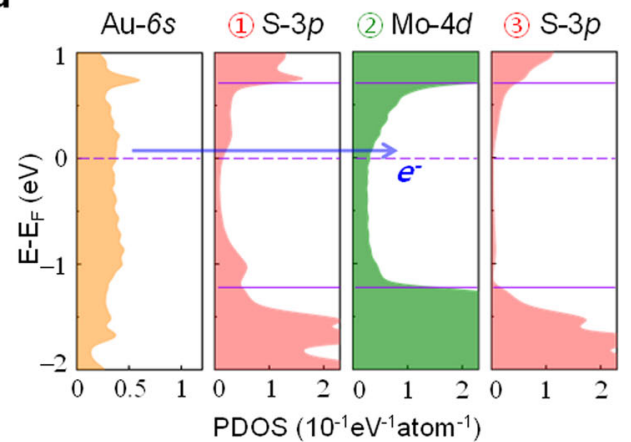

e

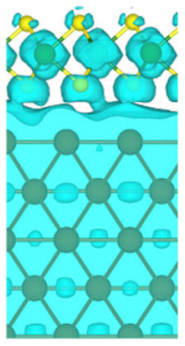

f

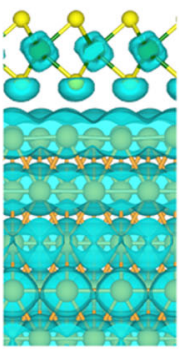

$E_{F}$ LDOS

Fig. 8 Atomistic origins of the field emission-dominated charge transport across In/MoS $\mathbf{S}_{\mathbf{2}}$ contacts. Projected band structures of the Mo- $4 d$ states (green-filled circle) for the $\mathbf{a} \mathrm{In} / \mathrm{MoS}_{2}$ and $\mathbf{b} \mathrm{Au} / \mathrm{MoS}_{2}$ interfaces. Shown together in $\mathbf{c}$ and $\mathbf{d}$ are the In-5p $p_{z}$ (pink filled circles) and Au-6s states (orange filled circles) contributions, respectively. The circle size is proportional to the weight of the projection of the wavefunctions. The PDOS of the In-5p (top left panel) and Au-6s (bottom left panel), (1) metal-contacting S-3p (second panels), (2) Mo-4d (third panels), and (3) outer

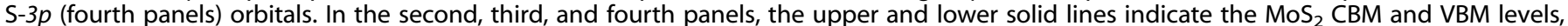
respectively. The arrows schematically show that the charge transport across the $\mathrm{In} / \mathrm{MoS}_{2}$ contact is much more efficient than that across the $\mathrm{Au} / \mathrm{MoS}_{2}$ counterpart, and the Ohmic-like charge transport is mediated by the large in-gap states formed around $E_{\mathrm{F}}$ of the In/MoS $\mathrm{S}_{2}$ interface. The local DOS in the range between $E_{\mathrm{F}}$ and $E_{\mathrm{F}}+0.2 \mathrm{eV}$ overlaid over the $\mathbf{e} \ln / \mathrm{MoS}_{2}$ and $\mathbf{f} \mathrm{Au} / \mathrm{MoS}_{2}$ interface models. The iso-surface level is $2.5 \times 10^{-4}$ states $\mathrm{eV}^{-1} \AA^{-3}$.

that of the specific contact resistivity within the field-emission region. For the monolayer $\mathrm{MoS}_{2}$ case, we achieved the contact resistance of $\sim 1 \mathrm{k} \Omega \mu \mathrm{m}$ at $T=100 \mathrm{~K}$, which is the lowest value achieved by metal evaporations on $\mathrm{MoS}_{2}$ to date. Our experimental findings were corroborated by DFT calculations, which showed that the In/MoS 2 contact has a weakened FLP due to the marginal interfacial charge transfer. Importantly, in spite of the weak interface dipole formation, we found that strong density of in-gap states are formed around $\mathrm{MoS}_{2} \mathrm{CBM}$ states and enable an Ohmic-like charge transport across the $\mathrm{In} / \mathrm{MoS}_{2}$ interface. Related with this identified mechanism, we comment that one of us previously predicted for semiconducting carbon nanotubes that a highly efficient charge injection across vdW metal contacts could be achieved via large in-gap states generated from topological defects within the $s p^{2}$ carbon network ${ }^{28}$. We thus suggest that seeking a mechanism of introducing strong density of in-gap states while maintaining the ideal contact geometry with weak charge transfer could prove to be a general strategy to simultaneously avoid FLP and minimize contact resistance for low-dimensional vdW materials ${ }^{42}$.

\section{METHODS}

\section{Device fabrication}

We fabricated $\mathrm{MoS}_{2}$ FETs on $h$-BN flakes, where the $h$-BN flakes were deposited onto a 300 -nm-thick $\mathrm{SiO}_{2} / \mathrm{Si}$ substrate by mechanical exfoliation. We then transferred a few-layer $\mathrm{MoS}_{2}$ flake (HQ-graphene, Inc.) onto a selected $h$-BN flake ${ }^{12,20}$. For the electrical measurements, we deposited 100-nm-thick In electrodes across the $\mathrm{MoS}_{2}$ channel, where the substrate holder was kept at $\sim 100 \mathrm{~K}$ by flowing liquid nitrogen through it. The substrate cooling process leads to an important result; a uniform surface morphology of In film is achieved, which contrasts strongly with the usage of a room-temperature holder that produces a segregated granular film for at least up to $\sim 70 \mathrm{~nm}$ thickness, as reported in our previous work (also see Supplementary Fig. 1$)^{21}$.

\section{Raman spectroscopy}

The Raman measurements were performed in a backscattering geometry at room temperature. An incident laser light with a wavelength of $514.5 \mathrm{~nm}$ was focused on the sample surface through an optical microscope objective lens $(100 \times / 0.9 \mathrm{NA})$. An excitation laser power was maintained less than $0.4 \mathrm{~mW}$ to avoid any laser-induced heating effects. Scattered light from the sample was dispersed through a monochromator with a 1200 grooves $\mathrm{mm}^{-1}$ grating and was collected using a thermoelectrically cooled charge-coupled device detector. For mapping measurements, Raman spectra were taken at the step of $0.5 \mu \mathrm{m}$ over the area of $15 \times 15 \mu \mathrm{m}^{2}$.

\section{DFT calculations}

We performed DFT calculations within the local density approximation $(\text { LDA })^{43}$ using the SIESTA software ${ }^{44}$. The atomic cores were replaced by norm-conserving nonlocal pseudopotentials of the Troullier-Martins type ${ }^{45}$, and double $\zeta$-plus-polarization-level numerical atomic orbital basis sets were employed. Geometry optimizations were performed until the Hellmann-Feynman ionic forces were below $0.02 \mathrm{eV} \AA^{-1}$. To check the reliability of geometries and electronic structures obtained within LDA in terms of vdW interactions, we also carried out DFT calculations using the DFT-D3 (ref. ${ }^{46}$ ) exchange-correlation functional. As in our earlier work ${ }^{47}$, we obtained consistent results from LDA and DFT-D3 calculations (see Supplementary Figs. 3, 4 and Table 1).

\section{DATA AVAILABILITY}

The datasets generated during and/or analyzed during the current study are available from the corresponding author on reasonable request. All data generated or 
analyzed during this study are included in this published article (and its Supplementary Information).

Received: 12 September 2020; Accepted: 25 November 2020; Published online: 08 January 2021

\section{REFERENCES}

1. Manzeli, S., Ovchinnikov, D., Pasquier, D., Yazyev, O. V. \& Kis, A. 2D transition metal dichalcogenides. Nat. Rev. Mater. 2, 17033 (2017).

2. Li, C., Zhou, P. \& Zhang, D. W. Devices and applications of van der Waals heterostructures. J. Semicond. 38, 031005 (2017).

3. Xia, F., Wang, H., Xiao, D., Dubey, M. \& Ramasubramaniam, A. Two-dimensional material nanophotonics. Nat. Photonics 8, 899 (2014).

4. Allain, A., Kang, J., Banerjee, K. \& Kis, A. Electrical contacts to two-dimensional semiconductors. Nat. Mater. 14, 1195 (2015)

5. Schulman, D. S., Arnold, A. J. \& Das, S. Contact engineering for 2D materials and devices. Chem. Soc. Rev. 47, 3037 (2018).

6. Das, S., Chen, H. Y., Penumatcha, A. V. \& Appenzeller, J. High performance multilayer $\mathrm{MoS}_{2}$ transistors with scandium contacts. Nano Lett. 13, 100 (2013).

7. Kim, C. et al. Fermi level pinning at electrical metal contacts of monolayer molybdenum dichalcogenides. ACS Nano 11, 1588 (2017)

8. Yang, L. et al. Chloride molecular doping technique on $2 \mathrm{D}$ materials: $\mathrm{WS}_{2}$ and $\mathrm{MoS}_{2}$. Nano Lett. 14, 6275-6280 (2014).

9. Wang, J. et al. High mobility $\mathrm{MoS}_{2}$ transistor with low Schottky barrier contact by using atomic thick $\mathrm{h}-\mathrm{BN}$ as a tunneling layer. Adv. Mater. 28, 8302 (2016).

10. Cui, X. et al. Low-temperature Ohmic contact to monolayer $\mathrm{MoS}_{2}$ by van der Waals bonded Co/h-BN electrodes. Nano Lett. 17, 4781 (2017).

11. Leong, W. S. et al. Low resistance metal contacts to $M_{0} S_{2}$ devices with nickeletched-graphene electrodes. ACS Nano 9, 869 (2015).

12. Cui, X. et al. Multi-terminal transport measurements of $\mathrm{MoS}_{2}$ using a van der Waals heterostructure device platform. Nat. Nanotechnol. 10, 534 (2015).

13. Kappera, R. et al. Phase-engineered low-resistance contacts for ultrathin $\mathrm{MoS}_{2}$ transistors. Nat. Mater. 13, 1128 (2014).

14. Liu, Y. et al. Approaching the Schottky-Mott limit in van der Waals metalsemiconductor junctions. Nature 557, 696 (2018).

15. Wang, J. et al. Low-power complementary inverter with negative capacitance 2D semiconductor transistors. Adv. Funct. Mater. 30, 2003859 (2020).

16. Kong, L. et al. Doping-free complementary $\mathrm{WS}_{2}$ circuit via van der Waals metal integration. Nat. Commun. 11, 1866 (2020).

17. Wang, Y. et al. Van der Waals contacts between three-dimensional metals and two-dimensional semiconductors. Nature 568, 70 (2019).

18. Kim, B.-K. et al. Genuine Ohmic van der Waals contact between indium and $\mathrm{MoS}_{2}$. Preprint at https://arxiv.org/abs/1904.10295 (2019).

19. Lau, C. S. et al. Quantum transport in two-dimensional $W_{2}$ with high-efficiency carrier injection through indium alloy contacts. ACS Nano 14, 13700-13708 (2020).

20. Dean, C. R. et al. Boron nitride substrates for high-quality graphene electronics. Nat. Nanotechnol. 5, 722 (2010).

21. Choi, D.-H. et al. Van-der-Waals-gap tunneling spectroscopy for single-wall carbon nanotubes. Carbon 113, 237 (2017).

22. Chakraborty, B. et al. Symmetry-dependent phonon renormalization in monolayer $\mathrm{MoS}_{2}$ transistor. Phys. Rev. B 85, 161403 (2012).

23. Somvanshi, D. et al. Nature of carrier injection in metal/2D-semiconductor interface and its implications for the limits of contact resistance. Phys. Rev. B 96, 205423 (2017).

24. Lloyd, D. et al. Band gap engineering with ultralarge biaxial strains in suspended monolayer $\mathrm{MoS}_{2}$. Nano Lett. 16, 5836 (2016).

25. Li, H. et al. Optoelectronic crystal of artificial atoms in strain-textured molybdenum disulphide. Nat. Commun. 6, 7381 (2015).

26. Hui, Y. Y. et al. Exceptional tunability of band energy in a compressively strained trilayer $\mathrm{MoS}_{2}$ sheet. ACS Nano 7, 7126 (2013).

27. Kim, Y.-H. \& Kim, H. S. Anomalous length scaling of carbon nanotube-metal contact resistance: an ab initio study. Appl. Phys. Lett. 100, 213113 (2012).

28. Kim, H. S., Lee, G. I., Kim, H. S., Kang, J. K. \& Kim, Y.-H. Intrinsically low-resistance carbon nanotube-metal contacts mediated by topological defects. MRS Commun. 2, 91 (2012).

29. Popov, I., Seifert, G. \& Tomanek, D. Designing electrical contacts to $\mathrm{MoS}_{2}$ monolayers: a computational study. Phys. Rev. Lett. 108, 156802 (2012).

30. Farmanbar, M. \& Brocks, G. First-principles study of van der Waals interactions and lattice mismatch at $\mathrm{MoS}_{2}$ /metal interfaces. Phys. Rev. B 93, 085304 (2016).
31. Tung, R. T. The physics and chemistry of the Schottky barrier height. Appl. Phys. Rev. 1, 011304 (2014).

32. Fivaz, R. \& Mooser, E. Mobility of charge carriers in semiconducting layer struc tures. Phys. Rev. 163, 743 (1967).

33. Kaasbjerg, K., Thygesen, K. S. \& Jacobsen, K. W. Phonon-limited mobility in n-type single-layer $\mathrm{MoS}_{2}$ from first principles. Phys. Rev. B 85, 115317 (2012).

34. Kim, S. et al. High-mobility and low-power thin-film transistors based on multilayer $\mathrm{MoS}_{2}$ crystals. Nat. Commun. 3, 1011 (2012).

35. English, C. D., Shine, G., Dorgan, V. E., Saraswat, K. C. \& Pop, E. Improved contacts to $\mathrm{MoS}_{2}$ transistors by ultra-high vacuum metal deposition. Nano Lett. 16, 3824 (2016).

36. Xia, F., Perebeinos, V., Lin, Y. M., Wu, Y. \& Avouris, P. The origins and limits of metal-graphene junction resistance. Nat. Nanotechnol. 6, 179 (2011).

37. Gong, C., Colombo, L., Wallace, R. M. \& Cho, K. The unusual mechanism of partial Fermi level pinning at metal-MoS 2 interfaces. Nano Lett. 14, 1714 (2014).

38. Guo, Y., Liu, D. \& Robertson, J. 3D behavior of Schottky barriers of 2D transitionmetal dichalcogenides. ACS Appl. Mater. Interfaces 7, 25709 (2015).

39. Liu, Y., Stradins, P. \& Wei, S. H. Van der Waals metal-semiconductor junction: Weak Fermi level pinning enables effective tuning of Schottky barrier. Sci. Adv. 2, e1600069 (2016)

40. Zhong, $\mathrm{H}$. et al. Interfacial properties of monolayer and bilayer $\mathrm{MoS}_{2}$ contacts with metals: beyond the energy band calculations. Sci. Rep. 6, 21786 (2016).

41. Kang, J., Liu, W., Sarkar, D., Jena, D. \& Banerjee, K. Computational study of metal contacts to monolayer transition-metal dichalcogenide semiconductors. Phys. Rev. X 4, 031005 (2014).

42. Leonard, F. \& Talin, A. A. Electrical contacts to one- and two-dimensional nanomaterials. Nat. Nanotechnol. 6, 773 (2011).

43. Ceperley, D. M. \& Alder, B. J. Ground state of the electron gas by a stochastic method. Phys. Rev. Lett. 45, 566 (1980).

44. Soler, J. M. et al. The SIESTA method for ab initio order-N materials simulation. $J$. Phys. Condens. Matter 14, 2745 (2002).

45. Troullier, N. \& Martins, J. L. Efficient pseudopotentials for plane-wave calculations. Phys. Rev. B 43, 1993 (1991)

46. Grimme, S., Antony, J., Ehrlich, S. \& Krieg, H. A consistent and accurate ab initio parametrization of density functional dispersion correction (DFT-D) for the 94 elements H-Pu. J. Chem. Phys. 132, 154104 (2010).

47. Lee, J. et al. Origin and control of polyacrylonitrile alignments on carbon nanotubes and graphene nanoribbons. Adv. Funct. Mater. 28, 1706970 (2018).

\section{ACKNOWLEDGEMENTS}

We acknowledge Prof. E. Hwang and Dr. J.-S. Park for fruitful discussion. This work was supported by the Korea Research Institute of Standards and Science (KRISS-2020-GP2020-0001) and the National Research Foundation of Korea (NRF) (Grant Nos. 2018R1A2A1A05078440, SRC2016R1A5A1008184, 2020R1F1A1075216, 2019R1A2C1003366, 2017R1A2B3009872, 2016M3A7B4024133, 2013M3A6B1078881, and 2017R1A4A1015400). Growth of hexagonal boron nitride crystals was supported by the Elemental Strategy Initiative conducted by the MEXT, Japan, Grant Number JPMXP0112101001, JSPS KAKENHI Grant Number JP2OH00354 and the CREST (JPMJCR15F3), JST. Computational resources were provided by the KISTI Supercomputing Center (KSC-2018-C2-0032).

\section{AUTHOR CONTRIBUTIONS}

M.-H.B., J.-J.K, and Y.-H.K. conceived the research project. K.W. and T.T. grew the bulk $h$-BN. D.-H.C. and B.-K.K. fabricated the devices. D.-H.C. performed the TEM analysis. B.-K.K. performed the electrical measurements and analyzed the data with M.-H.B. H.K. and H.R. performed the Raman spectroscopy. T.-H.K. and Y.-H.K. performed the DFT calculations. M.-H.B, B.-K.K, T.-H.K., Y.-H.K., and J.-J.K. wrote the manuscript. All authors discussed the results and commented on the manuscript. B.-K.K., T.-H.K., and D.-H.C. contributed equally to this work.

\section{COMPETING INTERESTS}

The authors declare no competing interests.

\section{ADDITIONAL INFORMATION}

Supplementary information is available for this paper at https://doi.org/10.1038/ s41699-020-00191-z.

Correspondence and requests for materials should be addressed to J.-J.K., Y.-H.K. or M.-H.B. 
Reprints and permission information is available at http://www.nature.com/ reprints

Publisher's note Springer Nature remains neutral with regard to jurisdictional claims in published maps and institutional affiliations.

Open Access This article is licensed under a Creative Commons Acc Attribution 4.0 International License, which permits use, sharing,
adaptation, distribution and reproduction in any medium or format, as long as you give appropriate credit to the original author(s) and the source, provide a link to the Creative Commons license, and indicate if changes were made. The images or other third party material in this article are included in the article's Creative Commons license, unless indicated otherwise in a credit line to the material. If material is not included in the article's Creative Commons license and your intended use is not permitted by statutory regulation or exceeds the permitted use, you will need to obtain permission directly from the copyright holder. To view a copy of this license, visit http://creativecommons. org/licenses/by/4.0/.

(c) The Author(s) 2021 